\title{
The analysis of the DPSK transmission carrier frequency of time- varying channel model of XCTD profiler
}

\author{
Yu Zheng, Guo-Min Song, Ying-Sheng Shang, Xiang-Yu Jin, Xiao-Rui Wang, Lei Tian \\ and Jun-Wei Liu \\ School of Electronics and Information Engineering, Tianjin Polytechnic University, \\ TianJin, 300387, China \\ E-mail: zhengyu@tjpu.edu.cn
}

\begin{abstract}
The distortion of transmission signal ,when passed through the channel of the Expendable Conductivity-Temperature-Depth (XCTD) profiler under the influence of the probing depth, transmission frequency, random noise and other factors, will be more serious. And it will affect the stability and accuracy of the DPSK transmission. This paper firstly establishes the model of XCTD channel and explains the theory of differential phase shift keying (DPSK) demodulation, which proposes that the selection of the demodulation carrier frequency is the key factor to affect the DPSK demodulation accuracy. Through specific analysis of the impact of the demodulation carrier frequency offset, we ascertain the best demodulation carrier transmission frequency satisfies the demand of the actual measurement within the range of detecting depth of 2000 meters. Meanwhile, we analyze that the increase of the probing depth and random noise based on the increase of transmission frequency has an influence on the size of bit error rate caused by the demodulation frequency offset. The results show that we can further optimize the design of the XCTD profiler measurement equipment, and improve the accuracy of the measurement data and the stability of the transmission signal.
\end{abstract}

Keywords: XCTD; Time-Varying Channel; DPSK Modulation; Transmission Carrier Frequency.

\section{Introduction}

The XCTD profiler measurement equipment is one of the most important instruments for deep-sea exploration [1-2]. The XCTD is single disposable goods. Additionally, the whole system for signal transmission is consist of the wired channel which has 2000 meters, and the single transmission line is the parallel enameled wire that diameter is about $0.1 \mathrm{~mm}$. The two spools twined by spiral inductance are respectively installed on the underwater probe and the internal of a launcher on board, and when the probe was launched into seawater by the launcher placed in the stern of the boat, the probe falls at a rate of 3 5 $\mathrm{m} / \mathrm{s}$ in water [3]. Meanwhile, the spool gradually unfolds with the probe sinking. 
The data collector in the internal of the probe accomplished acquisition, processing and transmission about marine environment parameters in real-time and the data transfer to the boat in the form of digital signal, and then form a metrical profile.

At present, XCTD mainly use the mode of the DPSK signal demodulation. Due to the increase of probing depth and transmission frequency reducing the extraction accuracy of carriers, the same frequency and phase of carrier will reduce the accuracy of the DPSK band transmission. Since the change of signal phase delay is relatively stable when we modulate the single by the highfrequency carrier during the process of despooling within the probing depth of 2000 meters ${ }^{[4]}$, which has a little influence on the stability of DPSK demodulation. This paper mainly analyzes the influence of the demodulation carrier transmission frequency to DPSK signal demodulation. Through a concrete analysis of the properties of transmission line, this paper calculates by the value of the impedance parameter and analyzes the influence of the demodulation carrier frequency offset to the DPSK signal demodulation in detail based on the channel model settled before. It shows that the demodulation carrier frequency offset have great significance for improving the signal transmission accuracy.

\section{The Establishment of the Impedance Model of the Channel and the Calculation of its Parameters}

The transmission channel of the XCTD profiler uses twin parallel enameled wire as the transmission line, and the two lines paralleled in the ocean. According to the results of previous research, the transmission lines wrapped around the spool are released, the length of the cable in the water increases and decreases in the two spools. The model of transmission channel is shown in Fig.1 (A) [5] and the transmission equation can be obtained as shown in formula (1-2).

$$
\begin{gathered}
H(S)=\frac{R_{0}}{b_{3} S^{3}+b_{2} S^{2}+b_{1} S^{1}+b_{0}} \\
\left\{\begin{array}{l}
b_{3}=4 L_{1} L_{2} C_{3} \\
b_{2}=2 C_{3}\left[L_{1}\left(R_{0}+2 R_{2}\right)+L_{2}\left(2 R_{1}+R_{3}+R_{4}\right)\right] \\
b_{1}=C_{3}\left[\left(2 R_{1}+R_{3}+R_{4}\right)\left(R_{0}+2 R_{2}\right)\right]+2\left(L_{1}+L_{2}\right) \\
b_{0}=R_{0}+2 R_{1}+2 R_{2}+R_{3}+R_{4}
\end{array}\right.
\end{gathered}
$$

The value of inductance, resistance of the spools and distributed capacitance between the transmission lines each other changes with the process of despooling. The variation of impedance parameters is shown in Fig.1 (B). 
As shown in Fig.1 (C), with the increase of the probing depth, the change of the transmission signal's phase will be greater, so it is more difficult for extracting the carrier signal.

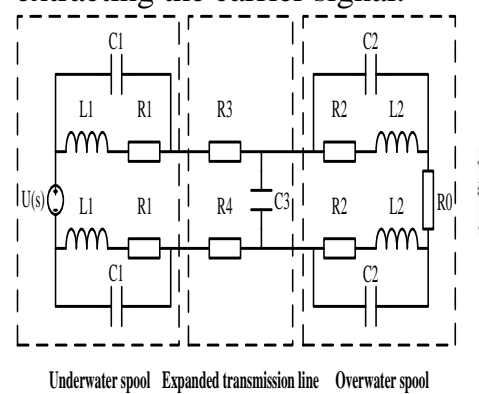

(A)

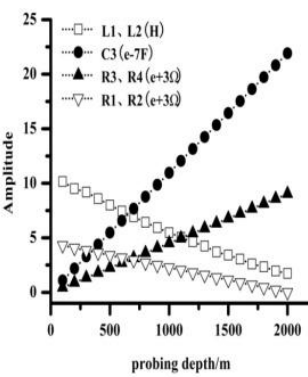

(B)

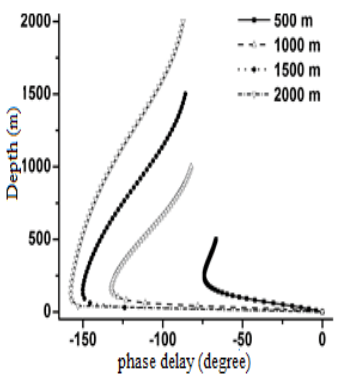

(C)

Fig. 1 the channel model and calculated result of impedance parameters (A)the channel's circuit model (B)the curves of impedance parameters (C)the diagrams between phase delay and depth

\section{The Principium of DPSK Modulation}

The principium of DPSK modulation is that binary PSK is a mode of digital modulation which the phase of carrier discretely changes with the binary digital baseband signal. In the DPSK modulation, as long as we keep the carrier phase of the before and after code element unchanged, the digital signal will not appear the problem of phase ambiguity after demodulation. The process of DPSK modulation and demodulation is shown in the Fig. 2.

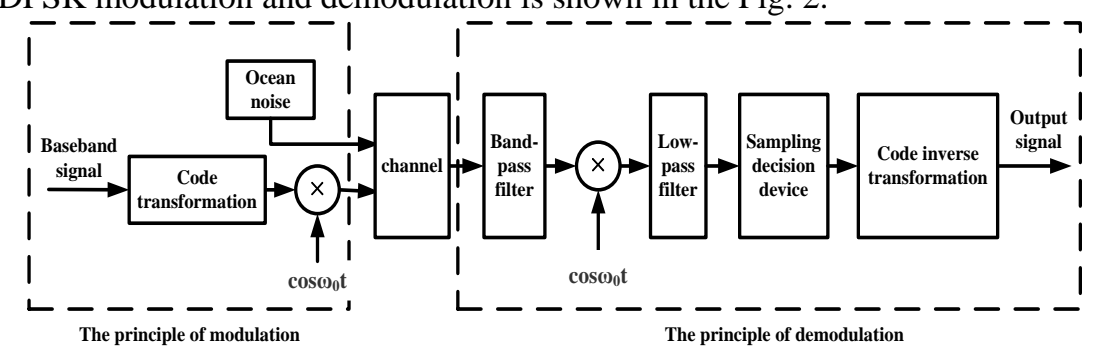

Fig. 2 the schematic diagram of DPSK transmission about the XCTD channel

In DPSK communication system, due to the differential coherent demodulation has poor performance in the aspect of resisting noise, we usually take coherence demodulation as the method to recover the primitive information. In the process, carrier same frequency is the key link which affects the accuracy of DPSK demodulation. The transmission channel of XCTD has a risk of timevarying property, which seriously affects the stability of the channel. This thesis analysis bit error rate caused by demodulation carrier frequency offset during the 
process of despooling within the probing depth of 2000 meters to ascertain the transmission frequencies of demodulation carrier.

\section{The Selection of Same Frequency Carrier in DPSK Demodulation}

According to the Modulation-Demodulation principle shown in Fig.2, we specifically analyze the size of bit error rate caused by carrier frequency offset during the process of DPSK demodulation by the different level of random noise and different range of probing depth. Therefore it can help us to select the transmission frequency of the demodulation carrier meeting the demand of actual measurement and clearly put forward the largest error range of the carrier frequency.

\subsection{The signal source's frequency is $300 \mathrm{~Hz}$}

Firstly, we specifically analyze the influence for increasing or decreasing the frequency of the demodulation carrier to DPSK demodulation in the process of despooling within $2000 \mathrm{~m}$ when we choose $300 \mathrm{~Hz}$ as the transmission frequency of signal source and $1500 \mathrm{~Hz}$ as the demodulation carrier frequency. The change of bit error rate caused by carrier frequency offset with the probing depth increasing is shown in Fig. 3 (a), and the change with the level of random noise increasing is shown Fig. 3 (b).
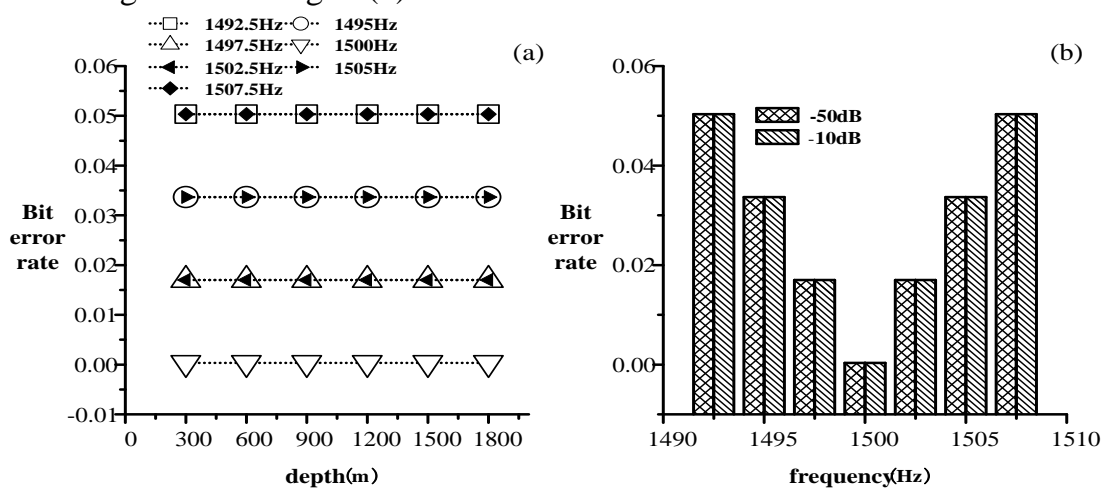

Fig. 3 the carrier frequency offset has an influence on DPSK demodulation when the transmission frequency of signal source is $300 \mathrm{~Hz}$ (a) increasing the probing depth (b) increasing the random noise

The analysis from the Fig.3 reveals that when the demodulation carrier frequency keeps the same with the modulation carrier frequency, the error rate is the minimum and the stability of DPSK frequency band transmission is highest at this very moment. Additionally, the greater the frequency offset, the larger the error rate when the frequency of signal source is lower. 


\subsection{The signal source's frequency is $1200 \mathrm{~Hz}$}

In the section, we make the frequency of signal source to increase to $1200 \mathrm{~Hz}$ and set the carrier frequency at $6000 \mathrm{~Hz}$. And then we specifically analyze the size of bit error rate caused by carrier frequency offset and what influence either the increase of probing depth or random noise has on DPSK demodulation.
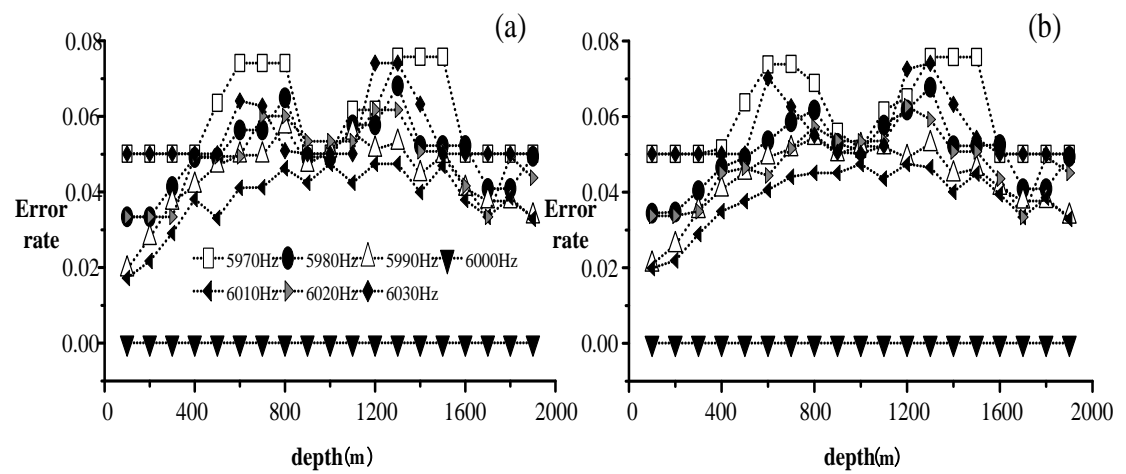

Fig. 4 the carrier frequency offset has an influence on DPSK demodulation when the transmission frequency of signal source is $1200 \mathrm{~Hz}$ (a) the level of random noise is $10^{-5} \mathrm{~dB}$ (b) the level of random noise is $10^{-1} \mathrm{~dB}$

The analysis from the Fig.4 reveals:(a) when the demodulation carrier frequency keeps the same with the modulation carrier frequency, the error rate is minimum. (b)the error rate will decrease with the frequency of signal source increasing when the carrier frequency offset is fixed. The increase of probing depth is the major factor of the change of the bit error rate. (c) the error rate is smaller in the mid-process of despooling and keep relatively stable at shortrange and great-depth measurement.

\section{Conclusion}

As a typical expendable conductivity temperature depth profiler measurement equipment, XCTD has an great significance on the ocean surveying. Because the channel parameters change dynamically in real-time, it has an great influence on the demodulation accuracy of the DPSK signal. In the paper, we make it clear that the best demodulation carrier frequency of the signal meeting the demand of actual measurement should keep same with the modulation carrier frequency. Moreover, we clearly put forward the error range of the extracted frequency of demodulation carrier. Neither the increase of probing depth nor random noise has no influence on the DPSK demodulation when the signal transmits with the 
best demodulation carrier frequency. However, the greater the carrier frequency offset, the greater the bit error rate. Meanwhile, the influence that the increase of probing depth or random noise have on the error rate with the signal source frequency increasing gradually increases during DPSK demodulation. So these conclusions have important significance to great-depth measurement of XCTD and solving the problem of the instability of XCTD channel transmission.

\section{Acknowledgments}

This research was supported by the Research Program of Application Foundation and Advanced Technology of Tianjin (No.14JCYBJC16300).

\section{References}

1. Sy, A., 1993. Field evaluation of XCTD performance. International WOCE Newsletter 14, 33-37.

2. Johnson, G. C., 1995: Revised XCTD fall-rate equation coefficients from CTD data[J]. Atmos. Oceanic Technol.12, 1367-1373.

3. Shoichi Kizua,Hiroji Onishib. Evaluation of the fall rates of the present and developmental XCTDs[J]. Deep-Sea Research,I55(2008):571 586.

4. Zheng Y, Gao Y, Fang J. The research methods of time-varying channel model of XCTD profiler[J]. Journal of Geophysics and Engineering, 2015,12(5):849-856.

5. Zheng $\mathrm{Y}$, Zhao X, Li J. Analysis of time-varying channel effects on transmission performance of deep-sea abandoned measuring instrument[J]. Acta phys. sin., 2014,04:69-76.

6. Zhang S H. 1994 IEEE Trans. Instrum. Meas.43 929.

7. Li Zun ji, Wang Lijun. Application of fuzzy control in ball mill process [J]. Journal of North China Electric Power University,1997,24(1):1- 5.

8. Yue Heng, Zhou Xiao jie, Wang Xin. A simulation system for intelligent decoupling control of coal-pulverizing systems with ball mill[J]. Journal of System Simulation, 2002,14(10):1340- 1343.

9. Donna M. Kocak, Howard Painter, Glenn Anderson. Anode Design and Analysis for an Undersea Cabled Observatory with a Seawater Ground[J]. OCEANS 2011, 2011,19(22):1-7. 